\title{
REPRESENTABLE DIVISIBILITY SEMIGROUPS
}

\author{
by BRUNO BOSBACH
}

(Received 18th January 1989)

To B. H. Neumann on the occasion of his 80 th birthday

\begin{abstract}
By a divisibility semigroup we mean an algebra $(S, \cdot, \wedge)$ satisfying (A1) $(S, \cdot)$ is a semigroup; (A2) $(S, \wedge)$ is a semilattice; (A3) $x(a \wedge b) y=x a y \wedge x b y$; (A4) $a \leqq b \Rightarrow \exists x, y: a x=b=a y$.

A divisibility semigroup is called representable if it admits a subdirect decomposition into totally ordered factors.

In this paper various types of representable divisibility semigroups are investigated and characterized, admitting a representation in general or even a special decomposition, like subdirect sums of archimedean factors, for instance.
\end{abstract}

1980 Mathematics subject classification (1985 Revision): 05.

\section{Introduction}

A lattice-ordered algebraic structure is called representable if it admits a subdirect decomposition into totally ordered factors of similar type. So, the question of representability is of central interest, and there is an abundance of contributions to this topic (cf. [4]). In particular one finds a dozen of criteria for lattice-ordered groups to be representable (cf. $[1,9,10]$ ), due to Lorenzen [15], Sik [18], Byrd [6], Fuchs (verbal remark, see [9]), and Conrad [9], none of which however works in the lattice-semigroup case.

As a matter of fact, a criterion for subdirect products of totally ordered factors has been missing for two decades since L. Fuchs stated his Problem 41 in [10], although it had been known for some twenty years (cf. [11]), that the subdirect products of totally ordered factors of a class of lattice-ordered algebras form a variety, see also [12].

Then, in 1984, an answer was given independently in [4] and [17] which even turned out to be of symptomatical character [4], telling that a lattice-ordered algebra is representable if and only if its linearily composed polynomials satisfy:

$$
p(a) \wedge q(b) \leqq p(b) \vee q(a)
$$

The proof has to be done via ideal-congruences, and this might be the reason for the solution being so late. A lattice-ordered group is considered as l-group, and not as lattice-g. So congruences are normal subgroups, and nothing else.

In this paper we study divisibility-semigroups, in order to simplify and to replace 
condition ( 0 ) by further equational and also by structural properties. This will lead to several representation theorems, the most interesting seeming to be that a divisibilitysemigroup is representable if and only if it satisfies:

$$
e a e \wedge f a f=(e \wedge f) a(e \wedge f)
$$

which was stated for lattice-groups by L. Fuchs (cf. [9]).

\section{Preliminary notions}

By a divisibility-semigroup we mean an algebra $(S, \cdot, \wedge)$ of type $(2,2)$ satisfying

(A1) $(S, \cdot)$ is a semigroup.

(A2) $(S, \wedge)$ is a semilattice.

(A3) $x(a \wedge b) y=x a y \wedge x b y$.

(A4) $a \leqq b \Rightarrow \exists x, y: a x=b=y a$.

Divisibility-semigroups are join-closed (with $(a \wedge b) a^{\prime}=a \Rightarrow b a^{\prime}=a \vee b$ ) and it turns out that the underlying lattice is distributive and that multiplication distributes over meet and join from the right and (by duality) from the left.

A divisibility-monoid is called (right) normal if it satisfies in addition:

$$
\forall a, b \exists a^{\prime}, b^{\prime}: a^{\prime} \wedge b^{\prime}=1,(a \wedge b) a^{\prime}=a,(a \wedge b) b^{\prime}=b
$$

In what follows we shall sometimes be concerned with distributive lattice-semigroups, i.e. lattice-semigroups satisfying the distributive laws mentioned above. They are called dldsemigroups in [16].

Let $S$ be a dld-semigroup. $a \in S$ is called positive if it satisfies $a s \geqq s \leqq s a$ for all $s \in S$. Obviously the set $S^{+}$of all positive elements of $S$ is closed w.r.t. $\cdot \wedge$, and $\vee . S$ itself is called positive if each of its elements is positive, i.e. if $S=S^{+}$. As usual $S^{+}$is called the cone of $S$.

In a divisibility-semigroup the elements $x, y$ of (A4) can always be taken from $S^{+}$ whence we tacitly shall suppose them to be positive whenever they are involved in calculations.

There is a most important rule of arithmetic.

Lemma 0.1. In a positive dld-semigroup we have:

$$
a \wedge b c=a \wedge a c \wedge b c=a \wedge(a \wedge b) c=a \wedge b(a \wedge c)
$$

Let $S$ be a $d l d$-semigroup and let $e a=a=a e$. Then $e$ is called a unit of $a$. The set of all units of $a$ is denoted by $E(a)$. If $S$ is even a divisibility-semigroup no $E(a)$ is empty and in addition one has: 
Lemma 0.2. [2]. Let $S$ be a dld-semigroup. Then each pair a,e with $e \in E(a)$ satisfies

$$
a=(e \wedge a)(e \vee a)=(e \vee a)(e \wedge a) \text {. }
$$

A divisibility-semigroup need not contain an identity element 1 . But, every divisibilitysemigroup $S$ admits a canonical smallest divisibility-semigroup extension $\Sigma$ formed by the set of all $(S, \wedge)$-endomorphisms of type $f h^{-1}$ with $f=i d$ or $f=f_{a}: x \rightarrow a x$ or $f=\bar{f}_{a}: x \rightarrow x \wedge a x$, and $h=\bar{f}_{b}$ with suitable elements $a, b$. This leads in $\Sigma$ to $\alpha=\beta \Leftrightarrow x \cdot \alpha=$ $x \cdot \beta\left(\forall x \in S^{+}\right)$. Important elements are the idempotents. More precisely we have:

Proposition 0.3. [2]. In a divisibility-semigroup the idempotents are central and positive.

A semigroup is called 0 -cancellative if it satisfies $a x=a y \neq 0 \Rightarrow x=y$ and $x a=$ $y a \neq 0 \Rightarrow x=y$.

Lemma 0.4. A divisibility-semigroup $S$ is 0-cancellative iff it satisfies

$$
a e=a \neq 0 \Rightarrow e=1 \text { and } e a=a \neq 0 \Rightarrow e=1 \text {, }
$$

since $a x=a y=a(x \wedge y) \Rightarrow a x=a(x \wedge y) x^{\prime}=a(x \wedge y) y^{\prime}=a y$.

A most important class of divisibility-semigroups is the class of archimedean divisibility-semigroups.

Definition 0.5. A divisibility-semigroup is called archimedean if it satisfies

$$
t^{n} \leqq a(\forall n \in \mathbb{N}) \Rightarrow t a t \leqq a
$$

In order that a divisibility-semigroup be archimedean it suffices that its cone is archimedean. Furthermore a fundamental result tells:

Theorem 0.6 [3]. Archimedean divisibility-semigroups are commutative.

We now turn to properties closely connected with representability, also called the vector property. Here, as an application of $(0)$, we get the criterion:

Proposition 0.7. [4] A lattice-semigroup is representable if and only if it is a dldsemigroup satisfying $x a y \wedge u b v \leqq x b y \vee$ uav where $x, y, u, v$ are taken from $S \cup\{1\}$.

For a divisibility-semigroup $S$ there is no need for an additional element 1 since there are always enough private units. Furthermore a commutative divisibility-semigroup is always representable. However, this fails to be true for dld-semigroups in general, consult [16], whereas commutative dld-monoids do have the vector property. 
Representability depends on the behaviour of certain substructures, the most important being lattice ideals.

Definition 0.8. Let $S$ be a $d l d$-semigroup. A nonempty subset $A$ of $S$ is called an ideal (filter) if it is an ideal (filter) of $(S, \wedge, \vee)$. An ideal (filter) $A$ is called irreducible if it cannot be written as intersection of two ideals (filters) different from $A$. An ideal $A$ is called $m$-ideal if it is multiplicatively closed. It is called invariant if it satisfies $x A=A x$. A filter $A$ is called Rees-filter if it satisfies $S \cdot A, A \cdot S \subseteq A$. Finally an ideal is called positive if it contains at least one positive element.

By definition $A$ is an irreducible ideal if $S-A$ is an irreducible filter. Furthermore it is folklore that an ideal (filter) $P$ is irreducible if and only if

$$
a \wedge b(a \vee b) \in P \Rightarrow a \in P \text { or } b \in P \text {. }
$$

Proposition 0.9. Let $S$ be a dld-semigroup. There are crucial congruences defined via ideals and filters, respectively.

(I) Let $P$ be an irreducible ideal (filter). Then $P$ generates a congruence via

$$
a \equiv b(P): \Leftrightarrow x a y \in P \leftrightarrow x b y \in P,
$$

where obviously $\equiv(P)$ is equal to $\equiv(S-P)$. Furthermore $S / P$ is totally ordered if in addition $S$ satisfies $(0)$.

(F) Let $R$ be a Rees-filter. Then $R$ generates a congruence via

$$
a \equiv b(R): \Leftrightarrow \exists x \in R: x \wedge a=x \wedge b .
$$

This implies that in the positive case every $x \in S$ generates a congruence mod $x$ by $a \equiv b(x) \Leftrightarrow x \wedge a=x \wedge b$ with $S / \equiv=: S_{x}$.

(M) Let $M$ be an m-ideal of $S^{+}$. Then $M$ generates a left congruence via

$$
a \equiv b(M): \Leftrightarrow \exists e, f \in M: a \leqq b e \text { and } b \leqq a f .
$$

For the sake of decomposition it is necessary to have enough congruences of a given type, in order to separate each pair $a, b$, and it is convenient that we may restrict ourselves to pairs $a<b$ in arbitrary lattice-semigroups and even to positive pairs $a<b$ in divisibility-semigroups. Furthermore, with respect to irreducible ideals, we may apply that there are enough regular ideals, i.e. ideals, maximal with respect to not containing a given element $a$, and that regular ideals are irreducible.

As a further important class of substructures we present:

Definition 0.10. Let $S$ be a divisibility-monoid. By a solid submonoid of $S$ we mean a submonoid $A$ whose cone $A^{+}$is an $m$-ideal of $S^{+}$and whose elements are exactly all 
$a b^{-1}$ with $a, b \in A^{+}, b$ invertible. A solid submonoid $P$ of $S$ is called a prime monoid of $S$ if it satisfies $A \cap B \subseteq P \Rightarrow A \subseteq P \vee B \subseteq P$ ( $A, B$ solid). $P$ is called regular if it is maximal with respect to not containing some given element $a$.

Obviously $S$ itself is solid and with a family $A_{i}$ of solid submonoids also its intersection is solid. Hence, every subset $M$ of $S$ generates a smallest solid submonoid $C(M)$, which in the case of a positive $M$ turns out to be equal to the set of all $x \leqq m_{1} \cdot \ldots \cdot m_{n}\left(m_{i} \in M\right)$. Furthermore in analogy to the l-group case we have the propositions:

Proposition 0.11. Let $S$ be a divisibility-monoid. Then the set of all solid submonoids forms a distributive lattice and in addition complex-multiplication distributes over meet and join. (For an idea consult [1]).

Proposition 0.12. Let $S$ be a divisibility-monoid. Then every direct decomposition of $S^{+}$ induces a direct decomposition of the whole in such a way that the direct factors of $S$ are the solid submonoids generated by the direct factors of $S^{+}$. (For an idea consult [4]).

In some theorems of this paper we are concerned with direct factors. For this reason we remark $u \perp v: \Leftrightarrow u \wedge v=1$.

Definition 0.13. Let $S$ be a divisibility-monoid, and let $A \subseteq S$. Then the polar $A^{\perp}$ of $A$ is defined by

$$
A^{\perp}:=\left\{x \mid \forall a \in A:(1 \vee a)(1 \wedge a)^{-1} \perp(1 \vee x)(1 \wedge x)^{-1}\right\}
$$

Furthermore the bipolar of $A$ is defined by $A^{\perp \perp}:=\left(A^{\perp}\right)^{\perp}$, and the polar of a singleton $\{a\}$ is also written as $a^{\perp}$, (compare [4]).

Proposition 0.14. Let $S$ be a divisibility-monoid. Then every polar is solid and moreover a solid submonoid $A$ is a direct factor if and only if $A \cdot A^{\perp}=S$, and in this case $A$ is equal to $A^{\perp \perp}$.

Finally we remark on some results which are proved straightforwardly—see also [1].

Lemma 0.15. Let $S$ be a normal divisibility-monoid. $P \subseteq S$ is a prime submonoid iff $P$ is solid and $a \wedge b=1 \Rightarrow a \in P$ on $b \in P$.

Lemma 0.16. Let $S$ be a normal divisibility-monoid. Then each prime submonoid of $S$ contains a minimal prime submonoid.

Lemma 0.17. Let $S$ be a normal divisibility-monoid. Then each minimal prime submonoid $M$ is canonically associated with an ultrafilter of $\left(S^{+}, \wedge, \vee\right)$ by $M \rightarrow S^{+} \backslash M$ which implies that each minimal prime submonoid $M$ of $S$ is of type $M=\left\{x^{\perp} \mid x \notin M\right\}$. 
Lemma 0.18. Let $S$ be a normal divisibility-monoid. Then each regular submonoid is a prime submonoid.

\section{Subdirectly irreducible divisibility semigroups}

There is not too much known about subdirectly irreducible divisibility-semigroups in general. In the finite case however the situation is a bit better.

We start with a description of the subdirectly irreducible homomorphic images of arbitrary distributive lattice ordered semigroups.

Proposition 1.1. If $S$ is a dld-semigroup and $S / \Theta$ is subdirectly irreducible, then $\Theta$ is generated by an irreducible ideal (filter).

Proof. Let $a<b$ be a critical pair. We choose an $\bar{a}$ containing, $\bar{b}$ avoiding regular ideal $\bar{M}$ of $\bar{S}:=S / \Theta$ with inverse image $M$ in $S$. Then $\bar{M}$ is irreducible in $\bar{S}$ whence $M$ is irreducible in $S$.

Furthermore

$$
\bar{x} \equiv \bar{y} \Leftrightarrow \bar{s} \bar{x} \bar{t} \in \bar{M} \Leftrightarrow \bar{s} \bar{y} \bar{t} \in \bar{M}\left(s, t \in S^{1}\right)
$$

provides a congruence relation on $\bar{S}$, which according to the subdirect irreducibility of $\bar{S}$ must be the equality relation.

On the other hand we have

$$
\bar{s} \bar{x} \bar{t} \in \bar{M} \Leftrightarrow \bar{s} \bar{y} \bar{t} \in \bar{M} \Leftrightarrow s x t \in M \Leftrightarrow s y t \in M\left(s, t \in S^{1}\right)
$$

which yields

$$
x \Theta y \Leftrightarrow x \equiv y(M)
$$

The next result concerns idempotents in subdirectly irreducible divisibility-semigroups.

Proposition 1.2. Let $S$ be a subdirectly irreducible divisibility-semigroup. Then $S$ contains at most two idempotents.

Proof. Let $S$ be subdirectly irreducible and let $u \in S$ be idempotent. We define

$$
a \rho b: \Leftrightarrow \exists s \in S: a \wedge s u=b \wedge s u
$$

and

$$
a \sigma b: \Leftrightarrow a u=b u \text {. }
$$

It is easily seen that both definitions provide a congruence, and furthermore we get 


$$
\begin{aligned}
a u=b y \Rightarrow s u \vee a & =u(s u \vee a) \\
& =u(s u \vee b)=s u \vee b .
\end{aligned}
$$

But from this follows:

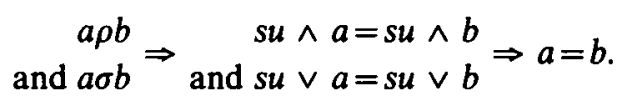

We now turn to the positive case, proving as a first general result:

Proposition 1.3. Let $S$ be a positive subdirectly irreducible dld-semigroup. Then in $S$ there exists a maximum 0 and a unique hyper-atom (co-atom) a which together form a critical pair.

Proof. Suppose that $a<b$ is critical. Then $x<b$ and $x \leq a$ implies $x \wedge a<x=x \wedge b$ whence $a$ and $b$ would be separated in $S_{x}$. Therefore we have $b=0$ and $x<b \Rightarrow x \leqq a$.

Applying 1.3 to the divisibility case we obtain in particular:

Proposition 1.4. Let $S$ be a positive subdirectly irreducible divisibility-semigroup. Then $S$ is a normal divisibility-monoid and hence totally ordered or containing an orthogonal pair $u^{*}, v^{*}$ with $1 \neq u^{*} \perp v^{*} \neq 1$. Verifying these properties it will turn out furthermore that the subset $L$ of all left cancellative elements and the subset $R$ of all right cancellative elements both form an irreducible m-ideal.

Proof. We start by proving the second assertion. We see immediately that the right and the left units of the hyper-atom $a$ form irreducible $m$-ideals because of $a x=a$ or $a x=0$. Furthermore we see that $e$ is a right unit of $a$ iff $e$ is right cancellative, since each right cancellative $c$ satisfies $a c \neq 0 c$ and since each right unit $e$ of $a$ produces a congruence separating $a$ and 0 , namely $x \equiv y \leftrightarrow x e=y e$.

Hence $L$ and $R$ form irreducible $m$-ideals and in addition every unit $e$ of $a$ is cancellative whence $S$ is a monoid.

Suppose now $u, v \leqq a$ and $(u \wedge v) u^{\prime}=u,(u \wedge v) v^{\prime}=v, u^{*}\left(u^{\prime} \wedge v^{\prime}\right)=u^{\prime}$ and $v^{*}\left(u^{\prime} \wedge v^{\prime}\right)=v^{\prime}$. Then $u^{*} \wedge v^{*}=1$ since $\left(u^{*} \wedge v^{*}\right)\left(u^{\prime} \wedge v^{\prime}\right)=u^{\prime} \wedge v^{\prime}$ and $(u \wedge v) u^{*}=(u \wedge v) u^{*}\left(u^{\prime} \wedge v^{\prime}\right)=u$ and $(u \wedge v) v^{*}=v$. Hence $u^{*} \wedge v^{*} \in R \cap L$ whence $S$ is normal on the grounds of rightleft-duality.

Definition 1.5. An ideal is called co-regular if it is a complement of a regular filter.

Obviously a co-regular ideal is irreducible and minimal within the set of all irreducible ideals containing a fixed element $a$. 
Proposition 1.6. For a positive dld-semigroup the subdirectlly irreducible homomorphic images correspond uniquely with the co-regular ideals; and thereby with the regular filters.

Proof. Let $J$ be co-regular with respect to $a$ and let $J$ not contain $b$. Then $\bar{a}$ is the uniquely determined hyper-atom in $\bar{S}:=S / J$, since otherwise $S \backslash J$ would not be maximal w.r.t. not containing $a$. Consider now a subdirectly irreducible homomorphic image $\bar{S}$ with $\bar{a} \neq \overline{0}$. Here $\{\overline{0}\}$ is the image of $\{\overline{0}\}$ and both $\{\overline{0}\}$ and $\{\overline{0}\}$ are regular filters with respect to the corresponding hyper-atoms. This means $\bar{S} \cong S / J \cong \bar{S}$. Hence $S / J$ is subdirectly irreducible.

The rest follows by 1.1. since the inverse image of a filter regular with respect to $\bar{a}$ is a regular filter with respect to $a$.

Proposition 1.7. Let $S$ be a commutative subdirectly irreducible divisibility-semigroup. Then $S$ is a totally ordered, 0-cancellative divisibility-monoid.

Proof. First of all $S$ is totally ordered (cf. the remark following 0.7). Let now $a<b$ be a positive critical pair. Then $S / E(a) \cong S$, whence $E(a)$ is a singleton, say $\{e\}$. We consider $x \leqq a$ and $x u=x$. Then $u \in E(a)$, i.e. $u=e$. Therefore $S$ is a monoid. It remains to verify that $a \leqq y=y u \neq 0$ implies $u=e$. But this follows since the set $F:=\{x \mid E(x) \neq E(a)\}$ is empty or forms a Rees-filter with $S / F \cong S$.

\section{Divisibility semigroups}

In this paragraph we give some structure theorems on representation.

Theorem 2.1. For a divisibility-semigroup $S$ the following are equivalent:

(i) $S$ is representable.

(ii) $x a y \wedge u b v \leqq x b y \vee u a v$.

(iii) $S^{+}$is representable.

(iv) $\Sigma^{+}$is representable.

(v) $a x \wedge y b \leqq a y \vee x b$.

(vi) eae $\wedge f a f=(e \wedge f) a(e \wedge f)$.

Proof. (i) $\Leftrightarrow$ (ii) is valid on the grounds of 0.7 .

(ii) $\Leftrightarrow$ (iii) is evident in one direction.

Assume now (iii) to be true and $S$ to be subdirectly irreducible. We consider

$$
x a y \wedge u b v, x b y \vee u a v .
$$

Obviously (ii) is true, iff for suitable elements $a^{\prime \prime}, b^{\prime \prime}$

$$
x a^{\prime \prime}(a \wedge b) y \wedge u b^{\prime \prime}(a \wedge b) v \leqq x b^{\prime \prime}(a \wedge \dot{b}) y \vee u a^{\prime \prime}(a \wedge b) v
$$

Therefore by 1.4 , (ii) is already valid if it is valid for all orthogonal pairs $a, b$. Furthermore, choosing suitable elements $x^{\prime}, u^{\prime}$, 


\title{
REPRESENTABLE DIVISIBILITY SEMIGROUPS
}

\author{
$x a y \wedge u b v \leqq x b y \vee u a v$
}

can be written as

$$
(x \wedge u) x^{\prime} a y \wedge(x \wedge u) u^{\prime} b v \leqq(x \wedge u) x^{\prime} b y \vee(x \wedge u) u^{\prime} a v
$$

Hence (ii) is already valid if it is valid for all orthogonal pairs $a \perp b, x \perp u$ from which it follows that (ii) is already valid if it is valid for all orthogonal pairs $x \perp u, a \perp b, y \perp v$.

But this means a fortiori that (ii) holds in all of $S$ if it satisfied in $S^{+}$.

(iii) $\Leftrightarrow$ (iv) is an immediate consequence of the fact that $\alpha$ and $\beta$ of $\Sigma$ are equal if and only if $x \cdot \alpha=x \cdot \beta$ for all $x \in S^{+}$. To verify this we apply the more general lemma which tells that any identity holding in $S^{+}$is also valid in $\Sigma^{+}$and which follows from the implication

$$
x e=x \Rightarrow x \cdot f\left(\alpha_{1}, \ldots, \alpha_{n}\right)=x \cdot f\left(\alpha_{1} e, \ldots, \alpha_{n} e\right)
$$

We continue by considering (ii), (v), (vi).

(ii) $\Rightarrow(v)$ is evident.

(v) $\Rightarrow$ (vi) follows from

$$
e a e \wedge f a f \leqq e a f \wedge e a f=e a f \text { and } f a f \wedge e a e \leqq f a e \wedge f a e=f a e
$$

since

$$
(e \wedge f) a(e \wedge f)=e a e \wedge e a f \wedge f a e \wedge f a f
$$

(vi) $\Rightarrow$ (ii). First of all it suffices to consider the positive case. Hence we may start from a positive subdirectly irreducible $S$ with hyper-atom $a$.

This leads to $L \subseteq R$ or $R \subseteq L$ and thereby to $C=L$ or $C=R$. To see this assume $L \nsubseteq R \nsubseteq L$. Then there exist an $e \in L \backslash R$ and an $f \in R \backslash L$. But this means

$$
e a=a=a f \text { and } a e=0=f a
$$

which leads to the contradiction

$$
a=(e \wedge f) a(e \wedge f)=e a e \wedge f a f=0
$$

So in any case $C$ turns out to be an irreducible $m$-ideal. In particular this means that $p \perp q$ implies $p \in C$ or $q \in C$.

On the other hand, by the proof of (iii) $\Rightarrow$ (i) we may confine ourselves to orthogonal pairs $x, u ; a, b ; y, v$. But this means that we may start from the special situation

$$
x \perp u, a \perp b, y \perp v \text { and } a \in C .
$$

To gain a further reduction we prove that we may assume in addition 


$$
(x \wedge a) \wedge y=1 .
$$

This can be shown as follows:

$$
x \wedge a \wedge y \wedge y b v=1 \text {, by (0.1.). }
$$

Suppose now $(x \wedge a \wedge y) x^{*}=x$ and $(x \wedge a \wedge y) a^{*}=a$ and $(x \wedge a \wedge y) y^{*}=y$. We get $x^{*} \wedge a^{*} \wedge y^{*}=1$ by $(x \wedge a \wedge y)\left(x^{*} \wedge a^{*} \wedge y^{*}\right)=(x \wedge a \wedge y) \in C$ (recall $a \in C$ ), and moreover we have

$$
x^{*} a^{*} y^{*} \wedge u b v=x a y \wedge u b v
$$

according to 0.1. (Observe $x \wedge a \wedge y \perp u b v$ ).

Hence

$$
\begin{aligned}
x^{*} a^{*} y^{*} \wedge u b v & \leqq x^{*} b y^{*} \vee u a^{*} v \\
\Rightarrow x a y \wedge u b v & =x^{*} a^{*} y^{*} \wedge u b v \\
& \leqq x^{*} b y^{*} \vee u a^{*} v \leqq x b y \vee u a v .
\end{aligned}
$$

Summarizing, we have obtained that we may restrict ourselves to the case

$$
x \perp u, a \perp b, y \perp v, a \wedge x \perp y \text { and } a \in C .
$$

So by symmetry it is enough to consider the three cases

$$
\text { (1) } x, y \in C \text { and (2) } x, v \in C \text { and (3) } u, v \in C \text {. }
$$

Before treating these cases we remark as follows. Let $d, g$ be orthogonal. Then

$$
c \in C \Rightarrow c d \wedge g c \leqq d c d \wedge g c g=c \Rightarrow c(d \wedge c * g c)=c \Rightarrow d \perp c * g c .
$$

Observe that $c * g c$ and $c g: c$ are uniquely determined because $c$ is cancellative. This leads, by duality, to the implication

$$
c \in C \Rightarrow(d \perp g \Rightarrow d \perp c * g c \text { and } d \perp c g: c)
$$

which means: if $d$ and $g$ are orthogonal and $c$ is cancellative then $g c$ is equal to $c s$ for some $s \perp d$ and $c g$ is equal to $t c$ with some $t \perp d$.

Now we are in the position to treat the cases (1) through (3).

Case (1). Since $x, y \in C$ we get by (v) and $(L)$ : 
$x a y \wedge u b v=a^{*} x y \wedge u v b^{*} \quad\left(\right.$ with $\left.a^{*} \perp b^{*}\right)$

$$
\begin{aligned}
& \leqq a^{*}(x y \vee u v) a^{*} \wedge b^{*}(x y \vee u v) b^{*} \\
& =x y \vee u v .
\end{aligned}
$$

Case (2).

$$
\begin{aligned}
x a y \wedge u b v & =x a y \wedge(u \wedge x a y) b(v \wedge x a y) \quad(0.1 .) \\
& =x y a^{*} \wedge(u \wedge x a y)(v \wedge x a y) b^{*} \quad\left(\text { with } a^{*} \perp b^{*}\right) \\
& \leqq(x y \vee u v) a^{*} \wedge(x y \vee u v) b^{*} \\
& =x y \vee u v .
\end{aligned}
$$

Case (3). First of all (v) implies $a^{2} \wedge x^{2}=a \cdot 1 \cdot a \wedge x \cdot 1 \cdot x=(a \wedge x)^{2}$, which leads by cancellation to $(x * a)(a: x) \wedge(a * x)(x: a)=1$. Hence $a * x$ and $a: x$ commute. Therefore we can calculate:

$$
\begin{aligned}
x a y \wedge u b v & =(x \wedge a)(a * x)(a: x)(a \wedge x) y \wedge u b v \\
& =(x \wedge a)(a: x)(a * x) y(x \wedge a) \wedge u v b^{*}\left(x \wedge a \perp y, b^{*} \perp a\right) \\
& \leqq(x \wedge a)(a: x)(x y \vee u v)(x \wedge a)(a: x) \wedge b^{*}(x y \vee u v) b^{*} \\
& =x y \vee u v,
\end{aligned}
$$

thus completing Case (3) and finishing the proof of 2.1.

In the preceding theorem representable divisibility-semigroups were characterized by equations. In a further theorem we shall describe representable divisibility-semigroups by special substructure-properties which can be done adequately by studying the cone or more generally by considering the positive case of a divisibility-monoid, since in the positive case $S$ is turned to a divisibility-monoid by merely adjoining an identity 1 .

Theorem 2.2. For a positive divisibility-monoid $S$ the following are equivalent:

(i) $S$ is representable.

(ii) If $J$ is a co-regular ideal then its kernel

$$
\operatorname{ker}(J):=\{x \mid s \cdot t \in J \Rightarrow s \cdot x \cdot t \in J\}
$$

is irreducible. 
(iii) If $J$ is a co-regular ideal the set of all $m$-ideals between $\operatorname{ker}(J)$ and $J$ forms a chain under inclusion.

(iv) If $J$ is a co-regular ideal and $x \in S$ then the subsets

$$
X^{\perp}:=\{y \mid x \wedge y \in \operatorname{ker}(J)\}
$$

and

$$
X^{\perp \perp}:=\left\{z \mid \forall y \in X^{\perp}: y \wedge z \in \operatorname{ker}(J)\right\}
$$

satisfy

$$
X^{\perp} \cup X^{\perp \perp}=S .
$$

(v) If $J$ is a co-regular ideal then the subsets $X^{\perp}$ and $X^{\perp \perp}$ satisfy

$$
X^{\perp} \cdot X^{\perp \perp}=S
$$

Proof. (i) $\Rightarrow$ (ii). If $S$ is representable then $S / J$ is totally ordered and thereby $\overline{1}$ is $\wedge$-irreducible. But $\operatorname{ker}(J)$ is the inverse image of $\overline{1}$. So $\operatorname{ker}(J)$ is irreducible, too.

(ii) $\Rightarrow$ (i). If $\operatorname{ker}(J)$ is irreducible then $\overline{1}$ in $S / J$ is $\wedge$-irreducible. Hence $S / J$ is totally ordered on the grounds of 1.4 .

(i) $\Rightarrow$ (iii). Let $J$ be a co-regular ideal. Then $S / J$ is subdirectly irreducible and hence normal by 1.4 .

Consider now two $m$-ideals $A$ and $B$ between $\operatorname{ker}(J)$ and $J$ with $a \in A \backslash B, b \in B$. Since $S / J$ is totally ordered $\operatorname{ker}(J)$ is irreducible. So, choosing orthogonal elements $a^{\prime}, b^{\prime}$ with $(a \wedge b) a^{\prime}=a$ and $(a \wedge b) b^{\prime}=b$ we get $a^{\prime} \wedge b^{\prime} \in \operatorname{ker}(J)$ which implies $b^{\prime} \in \operatorname{ker}(J)$ and thereby $(a \wedge b) b^{\prime}=b \in A \cap B$, whence $B$ is contained in $A$.

(iii) $\Rightarrow$ (i). On the grounds of (iii) the kernels of co-regular ideals are irreducible. Hence, all we have to show is that there are enough co-regular ideals. But this is evident since there are enough regular filters.

(i) $\Rightarrow$ (iv). Let $S$ be representable and let $J$ be a co-regular ideal. Then $S / J=: \bar{S}$ is totally ordered and $\bar{x}^{\perp} \cup \bar{x}^{\perp \perp}=\bar{S}$ which yields condition (iv).

(iv) $\Rightarrow$ (i). Let $\bar{S}$ be as above. Then the hyper-atom $\bar{a}$ belongs to $\bar{x}^{\perp}$ or to $\bar{x}^{\perp \perp}$ for each $\bar{x} \in \bar{S}$. But this means $\bar{x}=\overline{1}$ or $\bar{x}^{\perp}=\{1\}$. Consequently there cannot exist an orthogonal pair in $\bar{S}$ whence $\bar{S}$ is totally ordered. Therefore condition (i) holds because $S$ has enough co-regular ideals.

(i) $\Rightarrow$ (v). Conclude similarly to (i) $\Rightarrow$ (iv).

(v) $\Rightarrow$ (i). Assume $J$ to be a co-regular ideal of $S$ and $S / J=: \bar{S}$ not to be totally ordered. Then by (v) the hyper-atom $\bar{a}$ of $\bar{S}$ is a product of an orthogonal pair $\bar{x}, \bar{y}$ which leads to $\bar{x}^{2} \leqq \bar{a}, \bar{y}^{2} \leqq \bar{a}$ and thereby to the contradiction

$$
\bar{a}=\bar{x} \cdot \bar{y}=\bar{x} \vee \bar{y}=\bar{x}^{2} \vee \bar{y}^{2}=\bar{x}^{2} \bar{y}^{2}=\bar{a}^{2}=\overline{0} .
$$

This completes the final part and thereby the whole of the proof. 
We continue our investigation by studying special representable divisibilitysemigroups. To this end we give

Definition 2.3. A divisibility-semigroup $S$ is called real if it is embeddable in $\mathbf{R}:=\left(\mathbf{R}^{\infty},+, \min \right)$ or $\mathbf{E}:=\mathbf{R}^{\geq 0} /\{x \mid x \geqq 1\}$ or $\mathbf{E}:=\mathbf{R}^{\geq 0} /\{x \mid x>1\}$.

As is easily seen 1 is a maximum of $\mathbf{E}$ and a hyper-atom of $\mathbf{E}$ :

Definition 2.4. Let $S$ be a divisibility-semigroup and $J$ an ideal of $S$. $J$ is called really archimedean if it satisfies the implication:

$$
u \cdot t^{n} \cdot v \in J(\forall n \in \mathbf{N}) \text { and } a \cdot b \in J \Rightarrow a \cdot t \cdot b \in J .
$$

Let $S$ be as above and let $F$ be a filter. $F$ is called really primary if it satisfies:

$$
a \cdot t \cdot b \in F \Rightarrow a \cdot b \in F \text { or } u \cdot t^{n} \cdot v \in F(\exists u, v \in S, n \in \mathbf{N}) .
$$

Obviously an irreducible ideal is really archimedean iff its complement $S-J$ is a really primary filter.

Theorem 2.5. For a divisibility-semigroup $S$ the following are equivalent:

(i) $S$ is a subdirect product of real divisibility-semigroups.

(ii) $S$ is a subdirect product of totally ordered archimedean divisibility-semigroups.

(iii) Every principal ideal is the intersection of a family of really archimedean irreducible ideals.

(iv) Every principle filter is the intersection of a family of really primary filtres.

Proof. (i) $\Rightarrow$ (ii) is evident.

(ii) $\Rightarrow$ (i). Let $\bar{S}$ be totally ordered and archimedean. Then it is easily checked that every homomorphic image of $\bar{S}$ is totally ordered and archimedean, too. So $\bar{S}$ can be decomposed into 0-cancellative totally ordered archimedean divisibility-semigroups, i.e. according to Hölder [13] and Clifford [7] into subsemigroups of R' and E: Observe that subdirectly irreducible positive components have a hyper-atom.

(i) or (ii) $\Rightarrow$ (iii) and (iv). Let $S$ be a subdirect product of real divisibility-semigroups. Then for every pair $a<b$ there exists an index $i$ with $i(a)<i(b)$, and the ideal $P_{i}:=\{x \mid i(x) \leqq i(a)\}$ is irreducible and really archimedean. Similarly we see that the filter $F_{i}:=\{x \mid i(x) \geqq i(b)\}$ is irreducible and really primary. But this means that there are enough ideals and enough filters to verify (iii) and (iv).

(iii) $\Leftrightarrow$ (iv) is valid by Definition 2.4 .

(iii) or (iv) $\Rightarrow$ (i) and (ii). We start from (iii). Then $S$ is archimedean and hence commutative. Indeed, $t \in S^{+}$and $t^{n} \leqq a(\forall n \in \mathbf{N})$ and $a<a t$ would imply the existence of a really archimedean ideal $P$ with $a \in P$ and (thereby) $t^{n} \in P(\forall n \in N)$, but $a t \notin P$. 
Let now $P$ be an irreducible really archimedean ideal of $S$ and suppose $\bar{t}^{n} \leqq \bar{c}(\forall n \in N)$ in $\bar{S}:=S / P$. Then we get

$$
\left(c \cdot s \in P \Rightarrow t^{n} \cdot s \in P(\forall n \in \mathbf{N})\right) \Rightarrow(c \cdot s \in P \Rightarrow c t \cdot s \in P)
$$

which means $\bar{c} \cdot \bar{t}=\bar{c}$. Thus we get (iii) $\Rightarrow$ (ii) whence (iii) or (iv) $\Rightarrow$ (i) and (ii).

\section{Divisibility monoids}

Up till now we have considered divisibility-semigroups in general. Henceforth we shall consider divisibility-monoids.

This will enable us to apply notions, well-known from lattice-group theory, due to pioneers like Jaffard and Conrad (cf. [14] and [8]), and well discussed above all by Bigard, Keimel and Wolfenstein in [1].

Let $G$ be a lattice-group. Recall that a solid submonoid $V$ of $G$ is called a value of $a$ if $V$ is maximal with respect to not containing $a$. The set of all values of $a$ is denoted by $\operatorname{val}(a) . G$ is called finite-valued if each val $(a)(a \in G)$ is finite.

$G$ is called ortho-finite if each bounded orthogonal subset $\left\{a_{i} \mid i \in I\right\}$ of $G$ $\left(a_{i}=a_{j} \vee a_{i} \wedge a_{j}=1\right)$ is finite.

$G$ is called semi-projectable if it satisfies $(a \wedge b)^{\perp}=a^{\perp} \vee b^{\perp}(\forall a, b \in G)$. $G$ is called projectable if it satisfies $G=a^{\perp} \times a^{\perp \perp}(\forall a \in G)$. $G$ is called strongly projectable if it satisfies $G=C(a) \times C(a)^{\perp}(\forall a \in G)$. Observe: strongly projectable implies $C(a)=\dot{a}^{\perp \perp}$.

Obviously each of these notions is based merely on the divisibility-monoid language. Hence we may adopt them once an identity is present.

Theorem 3.1. For a divisibility-monoid $S$ the following are equivalent:

(i) $S$ is a direct sum of totally ordered divisibility-monoids.

(ii) $S$ is normal, finite-valued, and semi-projectable.

(iii) $S$ is ortho-finite and projectable.

Proof. (i) $\Rightarrow$ (ii) is obvious.

(ii) $\Rightarrow$ (iii). First of all each prime submonoid contains exactly one minimal prime submonoid. To see this, assume $P$ to be prime and $A, B$ to be minimal prime and contained in $P$. Then there are elements $a \in A \backslash B, b \in B \backslash A$ which yield an orthogonal pair $a^{\prime} \in A \backslash B, b^{\prime} \in B \backslash A$ such that $a^{\perp} \subseteq B$ and $b^{\perp \perp} \subseteq A$. But this would lead to

$$
S=\left(a^{\prime} \wedge b^{\prime}\right)^{\perp}=a^{\perp} \vee b^{\prime \perp}=P .
$$

So we get next that $S$ is ortho-finite since $1 \leqq a_{i} \leqq a(i \in I)$ implies: $I$ is finite or there exists at least one value $M$ containing $a_{j}^{\perp}$ and $a_{k}^{\perp}(j \neq k)$, a contradiction which is seen as above.

Now we show that any regular $M \in \operatorname{val}(a)$ is a unique value with respect to some $c$. To this end we start from the family $\left\{M_{i} \mid i \in I\right\}$ of all minimal prime submonoids of $S$, 
not containing $a$. This set is finite since each $M_{i}$ is uniquely associated with some $V_{i} \in \operatorname{val}(a)$. So we have $\left\{M_{i} \mid i \in I\right\}=\left\{M_{0}, M_{1}, \ldots, M_{n}\right\}$ with $M_{0} \subseteq M$ and $M_{i} \nsubseteq M$ $(1 \leqq i \leqq n)$. But this leads to some $a_{i} \in M_{i} \backslash M$ for each $i \in I$ whence $M$ turns out to be the unique value of $c:=a \wedge a_{1} \wedge \cdots \wedge a_{n}$.

Suppose finally $S \neq a^{\perp} \times a^{\perp \perp}$. Then $a^{\perp} \times a^{\perp \perp}$ is contained in some $M$ with $\{M\}=\operatorname{val}(c)$, and since $a^{\perp \perp}$ is equal to $\bigcap h^{\perp}\left(h \in a^{\perp}\right)$ there exists at least one $h^{\perp}$ not containing $c$ and hence contained in $M$. But this yields a contradiction, since by $h^{\perp} \supseteq a^{\perp \perp}$ we get $h \in h^{\perp \perp} \subseteq a^{\perp}$ which implies

$$
S \neq M \supseteq a^{\perp} \vee h^{\perp}=(a \wedge h)^{\perp}=S .
$$

So (ii) $\Rightarrow$ (iii).

(iii) $\Rightarrow$ (i). Suppose $a \in S^{+}$and assume $a^{\perp \perp}$ not to be totally ordered. Then there exists an $x$ in $a^{\perp \perp}$ with $\{1\} \neq x^{\perp \perp} \subseteq a^{\perp \perp}$, but $x^{\perp \perp} \neq a^{\perp \perp}$. This leads to

$$
a^{\perp \perp}=x^{\perp \perp} \cdot\left(x^{\perp} \cap a^{\perp \perp}\right) \text { by }(0.11)
$$

and thereby to $a=a_{1} \cdot a_{2}$ with $a_{1} \in x^{\perp \perp}$ and $a_{2} \in x^{\perp} \cap a^{\perp \perp}$.

We know already $a_{1} \perp a_{2}$. Now we show $a_{1} \neq a \neq a_{2}$. To this end suppose first $a_{1}=a$. This implies $x^{\perp \perp}=a^{\perp \perp}$, a contradiction. Suppose next $a_{2}=a$. This leads to the implication: $a \in x^{\perp} \Rightarrow a^{\perp} \supseteq x^{\perp \perp} \Rightarrow x \in a^{\perp} \cap a^{\perp \perp}$, once more a contradiction. Therefore the decomposition of $a$ is proper. So, continuing the decomposition procedure, after finitely many steps we arrive at $a=a_{1} \cdot a_{2} \cdot \ldots \cdot a_{n}$ with pairwise orthogonal elements $a_{i}$, generating totally ordered bipolars $a_{i}^{\perp \perp}$. Consider now two totally ordered bipolars $x^{\perp \perp} \neq y^{\perp \perp}$. Then $z \in x^{\perp \perp} \cap y^{\perp \perp} \Rightarrow z^{\perp \perp} \subseteq x^{\perp \perp} \cap y^{\perp \perp} \Rightarrow z^{\perp \perp}=\{1\}$, whence $z=1$. Therefore the family of all totally ordered $x^{\perp \perp}$ can be taken to realize a decomposition of $S$ in the sense of (i).

For the sake of a further representation theorem we give next:

Definition 3.2. A divisibility-monoid is called strongly archimedean if it satisfies:

$$
1<t \Rightarrow \exists n \in \mathbf{N}: t^{n} \geqq a
$$

Strongly archimedean divisibility-semigroups are totally ordered [5], and according to Hölder's and Clifford's results a (totally ordered) divisibility-monoid is strongly archimedean iff it is embeddable in $\mathbf{R}$ or $\mathbf{E}$ or $\mathbf{E}$ :

Now we are ready to present

Theorem 3.3. For a divisibility-monoid $S$ the following are equivalent:

(i) $S$ is a direct sum of strongly archimedean totally ordered divisibility-monoids.

(ii) The lattice of solid submonoids of $S$ is boolean. 
(iii) $S$ is orthofinite and strongly projectable.

Proof. (i) $\Rightarrow$ (ii) is nearly obvious.

(ii) $\Rightarrow$ (iii). If the lattice of solid submonoids is boolean then every solid submonoid is a direct factor. But furthermore $S$ is also ortho-finite, since $C(M)$ cannot be a direct factor if $M$ is an infinite set of pairwise orthogonal elements with $a \in S$ as an upper bound.

(iii) $\Rightarrow$ (i). We could apply 3.1. but we wish to give some deeper information. Since every $C(x)$ is a direct factor, $S$ satisfies $a, t \in S^{+} \Rightarrow \exists n \in \mathbf{N}: a \wedge t^{n}=a \wedge t^{n+1}$.

Furthermore $S$ is normal. To see this we start from $(a \wedge b) a^{\prime}=a$ and $(a \wedge b) b^{\prime}=b$ with $a^{\prime}, b^{\prime} \in S^{+}$. It follows $b^{\prime}=b_{1}^{\prime} b_{2}^{\prime}$ with $b_{1}^{\prime} \in C\left(a^{\prime}\right)$ and $b_{2}^{\prime} \in C\left(a^{\prime}\right)^{\perp}$. This provides $b_{1}^{\prime} \leqq a^{\prime n}$ for some suitable $n \in \mathbf{N}$ which leads to $b_{1}^{\prime}=b_{11}^{\prime} \cdot b_{12}^{\prime} \ldots \cdot b_{1 n}^{\prime}$ with $b_{1 i}^{\prime} \leqq a^{\prime} \wedge b^{\prime}(1 \leqq i \leqq n)$. Thus we get $(a \wedge b) b_{1}^{\prime}=a \wedge b$ and thereby $(a \wedge b) a^{\prime}=a$ and $(a \wedge b) b_{2}^{\prime}=b$ with $a^{\prime} \perp b_{2}^{\prime}$.

Suppose now $1<x, y<a^{n}$ and $x \leqq y \leq x$. Then there are orthogonal elements $x^{\prime}, y^{\prime} \notin\{1\}$ whence $C(a)$ has a direct decomposition, say $C\left(x^{\prime}\right) \times D$. This leads to $C(a)=C\left(a_{1}\right) \times$ $C\left(a_{2}\right)$ with $a_{1} \perp a_{2}$, and, by continuing the procedure, after finitely many steps to a direct decomposition $C(a)=X C\left(x_{i}\right)$ where the direct factors $C\left(x_{i}\right)$ are directly indecomposable and hence totally ordered. Recall now that the lattice of all solid submonoids is distributive. This yields uniqueness of $X C\left(x_{i}\right)$ whence there are only finitely many totally ordered $C(x)$ with $a \wedge x \neq 1$.

So, taking all totally ordered $C(x)$ we get a family of strongly archimedean components in the sense of (i).

\section{Hypernormal divisibility monoids}

We continue our studies by considering a class of special normal divisibility-monoids.

Definition 4.1. A divisibility-monoid is called hypernormal if it satisfies:

$$
\begin{aligned}
& x, y \in S^{+} \text {and } a x \wedge a y=a \Rightarrow \exists z \perp x: a y=a z \\
& x, y \in S^{+} \text {and } x a \wedge y a=a \Rightarrow \exists z \perp x: y a=z a .
\end{aligned}
$$

Lemma 4.2. A divisibility-monoid is already hypernormal iff it satisfies:

$$
\begin{aligned}
& e \in S^{+} \text {and } a e=a \leqq b \Rightarrow \exists x \perp e: b=a x \\
& e \in S^{+} \text {and } e a=a \leqq b \Rightarrow \exists x \perp e: b=x a .
\end{aligned}
$$

Proof. Assume $a x \wedge a y=a$ and $(x \wedge y) y^{\prime}=y\left(y^{\prime} \in S^{+}\right)$. Then $y^{\prime}$ can be replaced by an element $y^{*} \perp x \wedge y$. Hence $z:=y^{*} \wedge y$ satisfies $a z=a y(z \perp x)$.

The hypernormal divisibility-monoid might be something like an optimal common abstraction of boolean rings (distributive lattices with boolean intervals) and lattice- 


\section{REPRESENTABLE DIVISIBILITY SEMIGROUPS}

groups. To have a natural example not boolean and not group-like, consider a Bezoutring $R$ with identity. Here one has

$$
a x \mid a \Rightarrow a=a x y \text { and } a z=a(x y-1+x y z)
$$

whence the principal ideal semigroup of $R$ is a hypernormal divisibility-monoid.

Lemma 4.3. Let $S$ be a hypernormal divisibility-monoid and let $J$ be an invariant $m$-ideal of $S$. Then $J$ generates a congruence and $S / J$ is hypernormal, too.

Proof. $J$ generates a congruence. Assume now $\bar{a} \bar{u}=\bar{a} \leqq \bar{b}$ and $b=a \vee b$. Then $a u \leqq a e$ whence $a(u \wedge e)=a(u \wedge e) u^{\prime}\left(u^{\prime} \in S^{+}\right)$and thereby

$$
\begin{aligned}
b & =a(u \wedge e) x \\
& =a(u \wedge e) y^{\prime}\left(y^{\prime} \perp u^{\prime}\right) .
\end{aligned}
$$

Hence we get

$$
\bar{b}=\bar{a} \overline{\left((u \wedge e) y^{\prime}\right)} \overline{\left((u \wedge e) y^{\prime}\right)}=\bar{y} \perp \bar{u} .
$$

The rest follows by duality.

Obviously 4.3. implies that $S / J$ is 0 -cancellative if it is totally ordered. Now we are in the position to prove:

Theorem 4.4. For a positive hypernormal divisibility-monoid $S$ the following are equivalent:

(i) $S$ is representable.

(ii) $x a \wedge b x \leqq x(a \wedge b) \vee(a \wedge b) x$.

(iii) $a \wedge b=1 \Rightarrow x a \wedge b x=x$.

(iv) $x a^{\perp}=a^{\perp} x$.

(v) $a, b \in S$ and $x a \wedge b x=x \Rightarrow \exists c, d \in S: \begin{array}{lll}c \perp a \text { and } c x=b x \\ d \perp b & \text { and } x d=x a .\end{array}$

(vi) Each minimal prime submonoid of $S$ is invariant (cf. [6]).

(vii) Each regular invariant $m$-ideal $J$ of $S$ is prime (cf. [9]).

Proof. (i) $\Rightarrow$ (ii) $\Rightarrow$ (iii) is obvious.

(iii) $\Rightarrow$ (iv). Suppose $a \perp b$. It follows

$$
x a \wedge b x=x=x a \wedge x c=x(a \wedge c) .
$$


This implies $x c=x c^{*}$ with $c^{*} \perp c \wedge a$ whence $z=c^{*} \wedge c$ satisfies $z \perp a$ and $b x=x z$. Thus we get $a^{\perp} x \subseteq x a^{\perp}$ and, by duality, $x a^{\perp} \subseteq a^{\perp} x$.

(iv) $\Leftrightarrow(v)$. Suppose $x a \wedge b x=x$. One gets $b x=x u$ and thereby

$$
\begin{aligned}
x a \wedge b x=x & \Rightarrow x a \wedge x u=x \\
& \Rightarrow x u=x u^{*}\left(u^{*} \perp a\right) \\
& \Rightarrow b x=x u^{*}=c x(c \perp a) .
\end{aligned}
$$

So (iv) implies (v).

Let now (v) be valid and suppose $a \perp b$ and $x b=d x$. Then we get $x a \wedge d x=x$, whence by (v) there exists an element $c$ such that $a \perp c$ and $c x=d x=x b$. This means $x a^{\perp} \subseteq a^{\perp} x$, and, by duality, $a^{\perp} x \subseteq x a^{\perp}$. (vi).

(iv) $\Leftrightarrow$ (vi). Since each minimal prime submonoid is a union of polars (0.17.) (iv) implies

On the other hand, if (vi) is valid, then each $m$-ideal of $S$ separating $a$ and $b$ contains a minimal prime submonoid of $S$, invariant by (vi). Hence (vi) implies (i) and thereby (iv).

(iv) $\Leftrightarrow$ (vii). Observe that for invariant $m$-ideals $J$ condition (iv) is carried over from $S$ to $S / J$. To see this, assume $(a \wedge b) a^{\prime}=a,(a \wedge b) b^{\prime}=b, a^{\prime} \perp b^{\prime}$, and $\bar{a} \perp \bar{b}$. One gets

$$
\begin{aligned}
a \wedge b \in J \Rightarrow x b & =x(a \wedge b) b^{\prime} \\
& =c x(a \wedge b)\left(c \perp a^{\prime}\right)
\end{aligned}
$$

and thereby $\bar{x} \bar{b}=\bar{c} \bar{x}(\bar{a} \perp \bar{c})$.

But this means that $\bar{x} \perp \bar{y} \Leftrightarrow \bar{x}=\overline{1}$ or $\bar{y}=\overline{1}$ and consequently that $J$ is prime. Thus (iv) $\Rightarrow$ (vii).

On the other hand we have (vii) $\Rightarrow$ (i) $\Rightarrow$ (iv).

The preceding theorem shows how strong hypernormal divisibility-monoids seem to be. This is confirmed also by the next result, a modification of $[1,14.1 .2]$ :

Theorem 4.5. For a hypernormal divisibility-monoid $S$ the following are equivalent:

(i) Each $a \in S$ satisfies $S=C(a) \times C(a)^{\perp}$. (Actually any strongly projectable divisibilitysemigroup is hypernormal, see above).

(ii) $S$ is a subdirect product $\prod S_{i}(i \in I)$ of strongly archimedean factors, satisfying: $\forall f$, $g \in S^{+} \exists n \in \mathbf{N}: f(x)^{n} \geqq g(x)(\forall x \in \operatorname{supp}(f))$.

(iii) $\forall a, t \in S^{+} \exists n \in \mathbf{N}: a \wedge t^{n}=a \wedge t^{n+1}$.

(iv) Each prime $m$-ideal is minimal.

Proof. (i) $\Rightarrow$ (ii). By (i) we have (iii), whence $S$ is commutative. Therefore it suffices to 
prove that the factors $\bar{S}=S / P$ are strongly archimedean. But this follows from $\bar{t}^{n}<\bar{a}(\forall n \in \mathbf{N}) \Rightarrow \exists m:\left(\bar{t}^{m}\right)^{2}=\bar{t}^{m}$ since $S / P$ is 0 -cancellative for each prime submonoid $P$.

(ii) $\Rightarrow$ (iii) is evident.

(iii) $\Rightarrow$ (iv). Each prime submonoid $P$ contains a minimal prime submonoid $M$. Suppose $M \neq P$. Then there exists an $x \in S^{+} \backslash P$ satisfying in $S / M=: \bar{S}$ for every arbitrary $y \in P^{+}$

$$
\bar{x}>\bar{y}^{n} \geqq \overline{1}(\forall n \in \mathbf{N}) .
$$

But this leads to $\bar{y}=\overline{1}$ as above, which means $y \in M$, and thereby $P=M$.

(iv) $\Rightarrow$ (i). Suppose $C(a) \times C(a)^{\perp} \neq S$. Then (iv) implies that $C(a) \times C(a)^{\perp}$ is contained in some minimal prime submonoid $M$. But by 0.17 each minimal prime submonoid $P$ of $S$ is of type $P=U\left\{x^{1} \mid x \notin P\right\}$ (cf. [1]). This completes the proof by contradiction.

\section{A final remark}

Two natural questions remain unsettled in this paper, namely how to characterize direct products of totally ordered divisibility-monoids and how to characterize irreducible representations of divisibility-monoids. So it should be remarked that a solution of these problems will be given elsewhere in a context which would have extended this paper unduly.

The clue to these results is the fact that the whole of Chapter 4 and nearly all of Chapter 7 of Bigard-Keimel-Wolfenstein carry over to normal divisibility-semigroups.

\section{REFERENCES}

1. A. BIGARD and K. Keimel, Groupes et Anneaux Réticulés (Springer, Berlin-GöttingenHeidelberg-New York, 1977).

2. B. Bosbach, Schwache Teilbarkeitshalbgruppen, Semigroup Forum 12 (1976), 119-135.

3. B. Bosвach, Archimedische Teilbarkeitshalbgruppen und Quader-Algebren, Semigroup Forum 20 (1980), 319-334.

4. B. Bosbach, Lattice ordered binary systems, Acta. Sci. Math. 52 (1988), 257-289.

5. B. Воsвасн, Teilbarkeitshalbgruppen mit vollständiger Erweiterung, J. Algebra 83 (1983), 237-255.

6. R. D. BYRD, Lattice ordered groups (Thesis, Tulane University 1966).

7. A. H. Clifford, Naturally totally ordered commutative semigroups, Amer. J. Math. 76 (1954), 631-646.

8. P. F. Conrad, Some structure theorems for lattice-ordered groups, Trans. Amer. Math. Soc. 99 (1961), 212-240.

9. P. F. ConRad, Lattice ordered groups (Tulane University, Dpt. Math. 1970).

10. L. Fuchs, Teilweise geordnete algebraische Strukturen (Vandenhoeck und Ruprecht in Göttingen, 1966). 
11. L. Fuchs, On partially ordered algebras I. Coll. Math. 13 (1966), 116-130.

12. L. Fuchs, A remark on lattice ordered semigroups, Semigroup Forum 7 (1974), 372-374.

13. O. Holder, Die Axiome der Quantität und die Lehre vom Maß. Sächs. Ges. Wiss., Math. Phys. Cl. 53 (1901), 1-64.

14. P. Jaffard, Contribution à l'étude des groupes ordonnés. J. Math. Pure Appl. 32 (1953), 203-280.

15. P. LoRenzen, Über halbgeordnete Gruppen, Math. Z. 52 (1949), 483-526.

16. V. B. REPNITZKIi, On subdirectly irreducible lattice-ordered semigroups. Semigroup Forum 29 (1984), 277-318.

17. V. B. Repnitzkil, Varieties of lattice ordered semigroups (Diss. Cand., Acad. of Sc. of Moldavia, Kichinew 1984).

18. F. S̆IK, Über subdirekte Summen geordneter Gruppen. Czechoslovak Math. J. 10 (1960), 400-424.

Fachbereich 17, Mathematik

GESAMTHOCHSCHULE/UnIVERSITÄT

3500 KASSEL

West Germany 